\title{
The Effect of Temperature and Partial Melting on Velocity and Attenuation in a Simple Binary System ${ }^{1}$
}

\author{
Hartmut Spetzler and Don L. Anderson \\ California Institute of Technology, Seismological Laboralory \\ Pasadena, California 91105
}

\begin{abstract}
A possible explanation of the low-velocity, low- $Q$ zone in the upper mantle is partial melting, but laboratory data are not available to test this conjecture. As a first step in obtaining an idea of the role that partial melting plays in affecting seismic variables, we have measured the longitudinal and shear velocities and attenuations in a simple binary system that is completely solid at low temperatures and involves $17 \%$ melt at the highest experimental temperature. The system investigated was $\mathrm{NaCl} \cdot \mathrm{H}_{2} \mathrm{O}$. At temperatures below the eutectic the material is a solid mixture of $\mathrm{H}_{2} \mathrm{O}$ (ice) and $\mathrm{NaCl} \cdot 2 \mathrm{H}_{2} \mathrm{O}$. At higher temperatures the system is a mixture of ice and $\mathrm{NaCl}$ brine. In the completely solid regime the velocities and $Q$ change slowly with temperature. There is a marked drop in the velocities and $Q$ at the onset of melting. For ice containing $1 \% \mathrm{NaCl}$, the longitudinal and shear velocities change discontinuously at this temperature by 9.5 and $13.5 \%$, respectively. The corresponding $Q$ 's drop by 48 and $37 \%$. The melt content of the mixture at temperatures on the warm side of the eutectic for this composition is about $3.3 \%$. The abrupt drop in velocities at the onset of partial melting is about three times as much for the ice containing $2 \% \mathrm{NaCl}$; for this composition, the longitudinal and shear Q's drop at the eutectic temperature by 71 and $73 \%$, respectively. If these results can be used as a guide in understanding the effect of melting on seismic properties in the mantle, we should expect sharp discontinuities in velocity and $Q$ where the geotherm crosses the solidus. The phenomena associated with the onset of melting are more dramatic than those associated with further melting.
\end{abstract}

\section{INTRODUCTION}

Knowledge of the mechanical properties of multicomponent systems in the vicinity of their melting points is required in various geophysical problems. In particular, the behavior of seismic velocity and attenuation near the melting point is pertinent to discussions of the upper mantle low-velocity zone. In this study we have measured the velocity and attenuation of longitudinal and shear waves in the vicinity of the eutectic temperature in a simple dilute binary system. By varying the composition, we have been able to study the effects of temperature and partial melting. We chose dilute solutions so that we could investigate the region involving partial melting. In the system studied, $\mathrm{NaCl} \cdot \mathrm{H}_{2} \mathrm{O}$, the amount of melt could be changed simply by varying either the temperature or the initial concentration of $\mathrm{NaCl}$.

Previous studies of this sort have used pure materials or eutectic mixtures, both of which

Contribution 1528, Division of Geological Sciences, California Institute of Technology, Pasadena 91105. have sharply defined melting temperatures rather than a melting interval. Mizutani and Kanamori [1964] measured the compressional and shear velocities and the compressional wave $Q$ for an alloy consisting of $\mathrm{Pb}, \mathrm{Bi}, \mathrm{Sn}$, and $\mathrm{Cd}$. The velocities varied approximately linearly with temperature until $T / T_{m}$ was about 0.97 , at which point they decreased rapidly. The $P$ velocity dropped $20 \%$ upon melting, and the quality factor $Q$ dropped by about an order of magnitude. The $Q$ decreased very rapidly as the melting point was approached. Similar results were obtained by Pokorny [1965], who obtained a velocity drop of about $15 \%$ and a $Q$ drop of an order of magnitude as melting progressed. Again the mechanical properties started to anticipate the melting point at a $T / T_{m}$ of about 0.97 .

Both of the preceding studies used an ultrasonic pulse method with frequencies in the high kilocycle or megacycle range, i.e., very short wavelengths. The actual amount of melt as a function of temperature and the configuration of the molten zones was not described.

Water and $\mathrm{NaCl}$ form a simple binary system 
that can easily be studied in the vicinity of the eutectic temperature. Both the phase diagram and the geometry of the components are well known. The melt phase (brine) occurs at the grain boundaries in cylindrical channels or thin layers, depending on the temperature. In polycrystalline specimens the melt occurs in irregularly shaped pockets between crystal and subcrystal boundaries. We used a resonance technique and large samples to assure that the wavelengths were always large compared with crystal or melt zone dimensions. Ice rods were frozen from dilute $\mathrm{NaCl}$ solutions, and the resonant frequencies were measured to obtain longitudinal and shear velocities. The quality factors, $Q$ 's, were obtained by measuring the width, and in some cases the decay, of the resonance peaks. The measurements were performed on pure $\mathrm{H}_{2} \mathrm{O}$ ice and on $\mathrm{NaCl}$-ice mixtures as a function of $\mathrm{NaCl}$ concentration and temperature for the fundamental mode and several overtones.

\section{Experimental Procedure}

The experiments were performed in a So-Low Environmental Equipment Co, refrigerator. To assure temperature stability the refrigerator was packed with ice, and the experiment was placed inside a styrofoam insulating box.

A carefully measured amount of distilled water was heated close to its boiling point, and the appropriate quantity of $\mathrm{NaCl}$ was added. To remove entrapped air, the solution was placed in an airtight container and a vacuum (10 torr) was pumped until the solution was boiling slowly. The solution was pumped under vacuum into a Teflon tube $2.54 \mathrm{~cm}$ ID and $30.5 \mathrm{~cm}$ long. The ultrasonic transducers were supported with Teflon plugs at each end of the tube. To avoid separation of the ice crystals from the brine during the freezing process, the samples were frozen quickly at about $-30^{\circ} \mathrm{C}$ and were rotated at $1 \mathrm{rpm}$ while in a horizontal position. The Teflon tubes were removed when the freezing was complete. After the resonance experiments were performed, the ice rods were melted and the salinity of the solution was remeasured. The salt content of the rod was approximately $10 \%$ less than the starting solution owing to concentration of salt at the surface of the rod during the early stages of freezing. Salinity was measured on one ice rod as a function of position within the rod. There was a slight concentration of salt toward the center of the rod and away from the ends. The tot variation, however, was less than $\pm 0.15 \%$ salt concentration.

Photomicrographs of thin sections cut fron. the ice rods were taken in order to study the size and orientation of the ice platelets and the distribution of the brine. From the photomicrographs it was possible to determine the size and orientation of the ice platelets. Most. platelets were oriented such that their $c$ axes were in planes parallel to the surface of the rod, Figure 1 shows a radial cut close to one end of a $2 \% \mathrm{NaCl}$ ice rod. The view is parallel to the $c$ axis of the platelets. The temperature was $-5.3^{\circ} \mathrm{C}$. The hexagonal platelets have an average diameter of $0.5 \mathrm{~mm}$, and their diameter-tothickness ratio is about 8 to 1 . Photographs of $1 \%$ ice $\mathrm{NaCl}$ show platelet diameters of approximately $1 \mathrm{~mm}$.

A solenoid arrangement was used to excite longitudinal modes in the ice. A small bar magnet was frozen into each end of the ice ror and the external transducer coils were enclose in aluminum boxes that were covered with metal to avoid electromagnetic coupling betw the driving and receiving transducers. transducers at each end of the ice rod wer identical and arranged symmetrically. The sheas transducers consisted of small flat coils which were frozen into the ice at both ends. To reduec electromagnetic coupling between the coils, the were oriented $90^{\circ}$ to each other. Permanent magnets were used at the driver and receiver end to complete the motor and dynamo action, respectively. The ice rods were supported by two narrow copper-band slings at the nodal points of the second harmonic. Various othe supports were tried, including three slings and foam rubber pads, but the best reproducibility and the highest $Q$ values were obtained with the two-sling arrangement, which was maintained throughout all measurements.

To monitor the temperature of the specimen, a separate ice rod was prepared under identical conditions to the one used for the velocity measurements. This control rod contained one thermocouple in the center and one on the ontside. By connecting the constantan of the tw iron-constantan couples, it was possible record the absolute temperature and the dit ence temperature between the outside and 


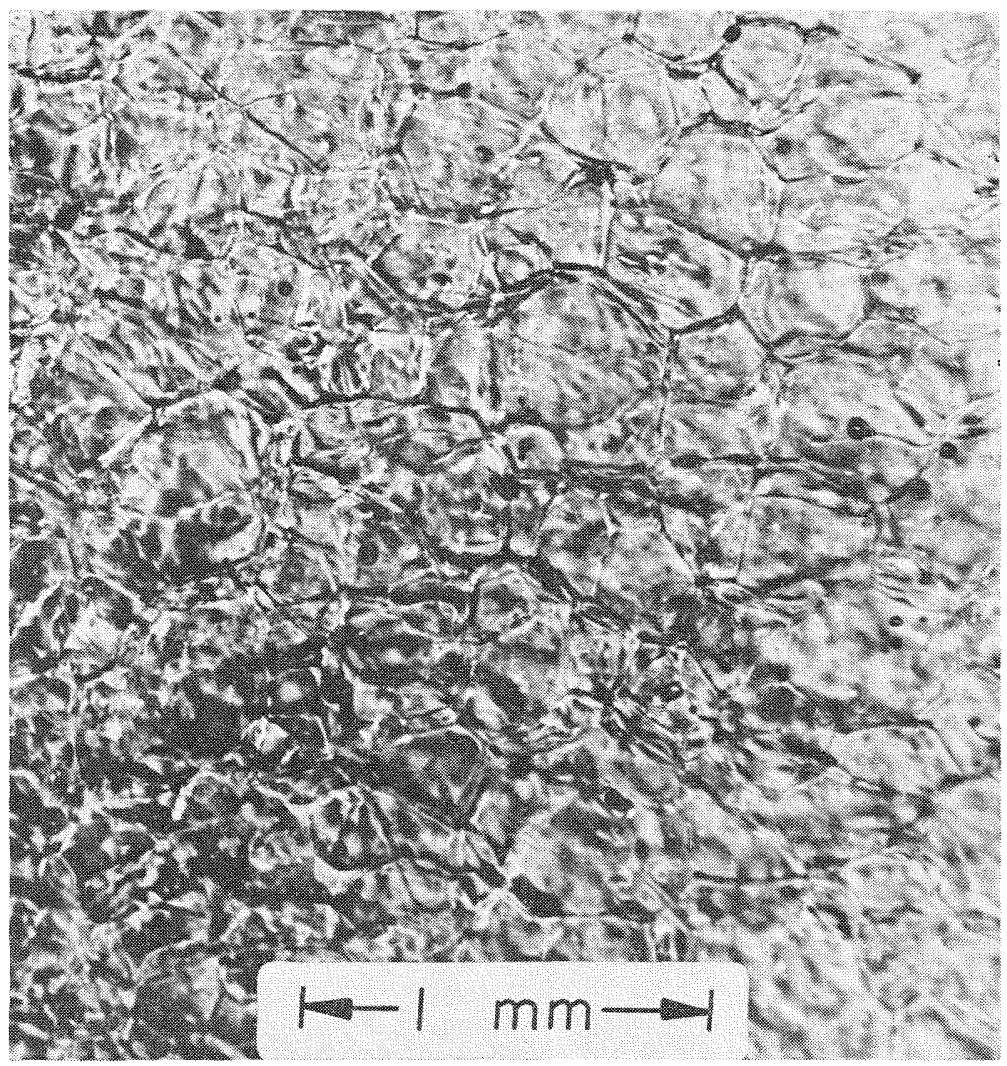

Fig. 1. A view parallel to the $c$-axis orientation of the ice platelets in ice containing $2 \%$ $\mathrm{NaCl}$.

side of the rod. The resistance between the top and the center and the center and the bottom of the rod was also measured. This measurement was performed to check for a possible brine drainage in the region warmer than the eutectic. No settling of brine was recorded n the time scale of the experiment. The absoute temperature was measured with the aid of a Leeds and Northrup potentiometer. Small temperature increments were read with a digital voltmeter. Periodic cross calibration between the voltmeter, the potentiometer, and various thermocouples suggests an absolute accuracy in temperature of $\pm 0.15^{\circ} \mathrm{C}$ and a relative accuracy between measurements of $\pm 0.05^{\circ} \mathrm{C}$. The reference ice bath was aerated and carefully maintained by using distilled water and shaved ice.

\section{INSTRUMENTATION}

1gure 2 shows the circuitry used to measure 1) record the resonant frequencies and the $Q$ 's of the various modes of the ice rods. A Schomandle ND30M frequency synthesizer was driven by a synchronous motor to sweep through the appropriate frequency range. To excite the Iongitudinal modes, the output of the synthesizer was amplified and applied directly to the driving solenoid.

The shear transducer arrangement, shown in Figure 2, is somewhat more complicated. An arrangement of resistors between synthesizer and amplifier served to select amplitudes for various frequency ranges. This arrangement enabled the operator to perform all measurements without disturbing the output level of the synthesizer. The output of the receiving transducer was amplified in two stages and then detected. The detected signal was recorded on a strip chart recorder and on a digital voltmeter. Both the driving signal and the output signal were displayed on an oscilloscope.

A typical record of a frequency sweep is 


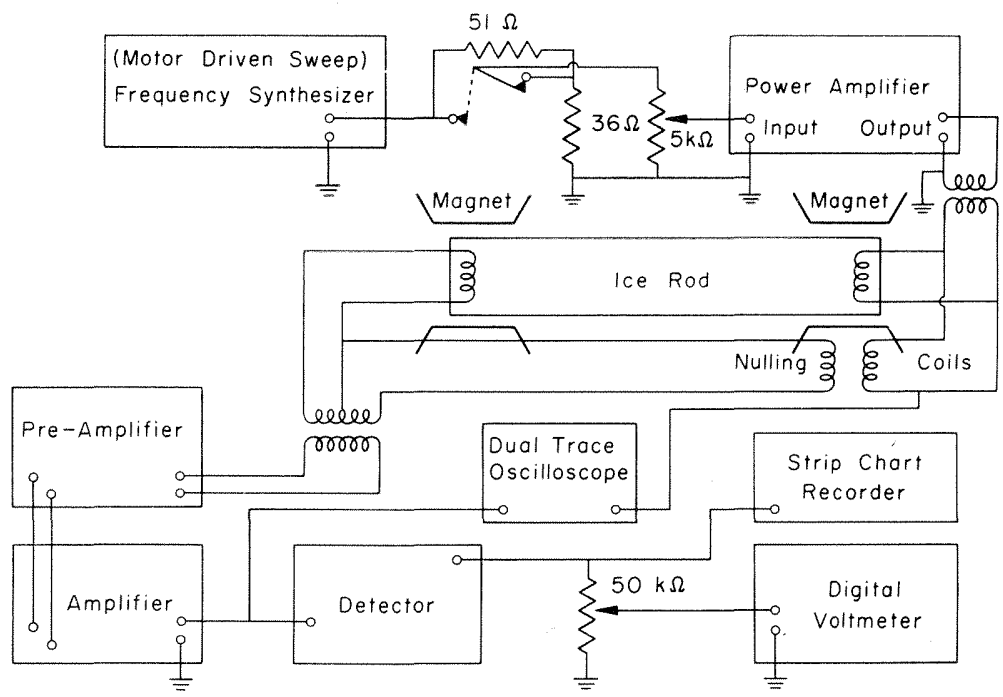

Fig. 2. Schematic illustration of the shear resonance experiment.

shown in Figure 3. The frequency at the peak of the resonance was read from the synthesizer. The widths of the symmetric resonance peaks were measured by changing the frequency to the values where the digital voltmeter was 0.707 of the peak value, thus giving the half-energy points. In cases of high noise level the resonance width was measured from the graph. Many data points for $Q$ were checked by measuring $Q$ from the decay of the rod oscillations after the power was turned off. The results were compatible.

A few data points were obtained on rods that were frozen under identical conditions in order to check reproducibility. These $Q$ and velocity data fell within the scatter of the data shown.

The perpendicular orientation of the sheartransducer coils did not completely eliminate the electromagnetic coupling between driver and receiver. To cancel the coupling, a second driver and receiver coil were placed outside the refrigerator and adjusted so that the electromagnetic coupling was the same as that for the coils embedded in the ice. The two receiver coils were connected to a center trapped transformer. By tuning off the resonance and adjusting the coupling of the compensating colls, the output of the two receiver coils could be made to be $180^{\circ}$ out of phase and thus cancel. The transformer served also to match the low impedance of the coils to the high input impedance of the amplifier. On the driver side, a transformer was used to match the impedance between the amplifier and the transducer coil.

\section{Experimental Results}

Measurements of resonant frequency and peak width were made for the fundamental and

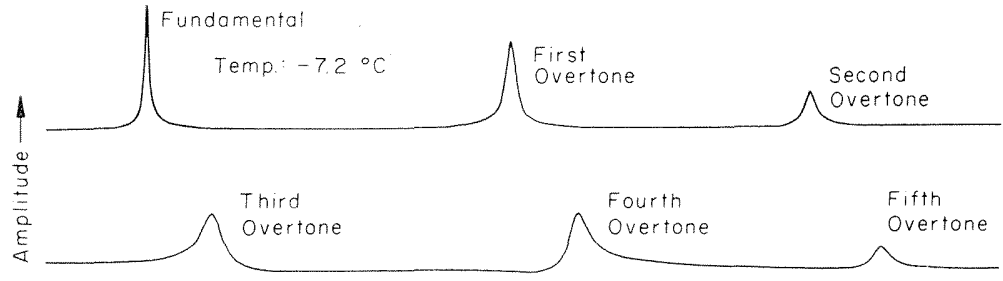

LONGITUDINAL MODES, PURE ICE

Fig. 3. Tracing of strip chart recording of a typical frequency sweep. 
several harmonics as a function of temperature. The temperature was varied slowly so that the difference temperature between the center of the rod and the outside never exceeded $0.1^{\circ} \mathrm{C}$. This required a cooling or heating rate between 1 and 2 degrees per hour, slower in the eutectic region. A typical data run from $-35^{\circ}$ to $-8^{\circ} \mathrm{C}$ and back through the eutectic took approximately 100 hours. The velocities were calculated from the fundamental resonance frequencies of the longitudinal and shear modes of the ice rods $V=2 l f / n$ where $f$ is the fundamental frequency of the appropriate mode, $n$ is the mode number, and $l$ is the length of the ice rod. No correction was applied for the temperature dependence of l. According to estimates of Weeks [1961], the maximum length change in the temperature from $-30^{\circ}$ to $-10^{\circ} \mathrm{C}$ for the $2 \% \mathrm{NaCl}$ ice would be approximately $0.3 \%$.

Velocity data from the fundamental mode of the 1 and $2 \%$ salt-ice-rods are presented in Figure 4 as a function of temperature. The eutectic temperature for the $\mathrm{NaCl} \cdot \mathrm{H}_{2} \mathrm{O}$ system is $-21.3^{\circ} \mathrm{C}$. Figure 5 gives the same data as a function of brine content. At temperatures colder than the eutectic, the velocity decreases approximately linearly as the temperature increases and at a faster rate than pure ice. In these experiments the absolute temperature was always greater than 0.94 of the eutectic temperature.

The normalized temperature derivatives of the velocities $-(1 / V) d V / d T$ are $0.6 \times 10^{-4}$, $0.9 \times 10^{-3}$, and $1.4 \times 10^{-3}{ }^{\circ} \mathrm{C}^{-1}$ for longitudinal waves in pure ice and $1 \% \mathrm{NaCl}$ and $2 \% \mathrm{NaCl}$ ice, respectively, for temperatures colder than the eutectic. The corresponding shear velocity derivatives are $0.8 \times 10^{-3}, 1.4 \times 10^{-3}$, and $2.8 \times 10^{-3}{ }^{\circ} \mathrm{C}^{-1}$, significantly larger than the longitudinal derivatives. The drop in longitudinal velocity across the eutectic for the ice containing 1 and $2 \% \mathrm{NaCl}$ was 9.5 and $28 \%$, respectively. The corresponding drops for the shear velocities were 13.5 and $40 \%$. These velocity decreases occur within $0.1^{\circ} \mathrm{C}$ but over a time span of several hours when the ice is warming up. When the ice is cooled through the eutectic point, the system is able to supercool by $1.5^{\circ}$ to $2.5^{\circ} \mathrm{C}$. In this case the eutectic region is spread over a larger temperature range but occurs in a shorter time interval. While the ice warms up, the velocities increase for several degrees immediately after the eutectic has been passed. When the temperature is reversed, the velocities do not show this dip.

Figure 5 gives the velocity as a function of brine content and salinity. To achieve a given brine content, the ice containing $1 \% \mathrm{NaCl}$

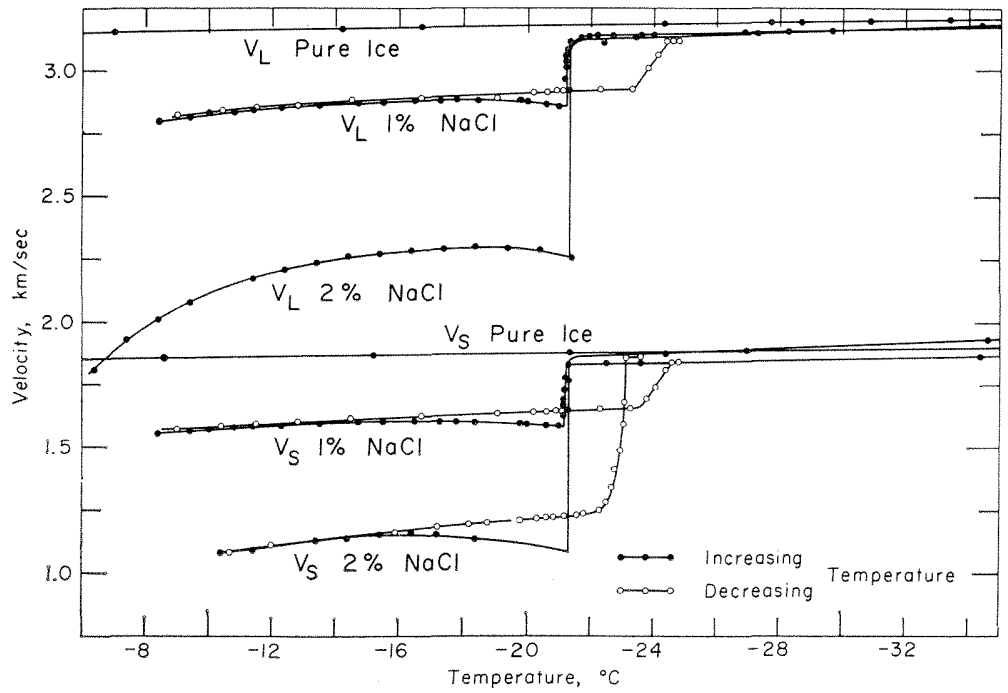

Fig. 4. Longitudinal $V_{L}$ and shear $V_{S}$ velocities in pure ice, ice containing $1 \% \mathrm{NaCl}$, and ice containing $2 \% \mathrm{NaCl}$. Note the large drop in velocity at the eutectic temperature and the hysteresis between the warming and cooling cycles. 


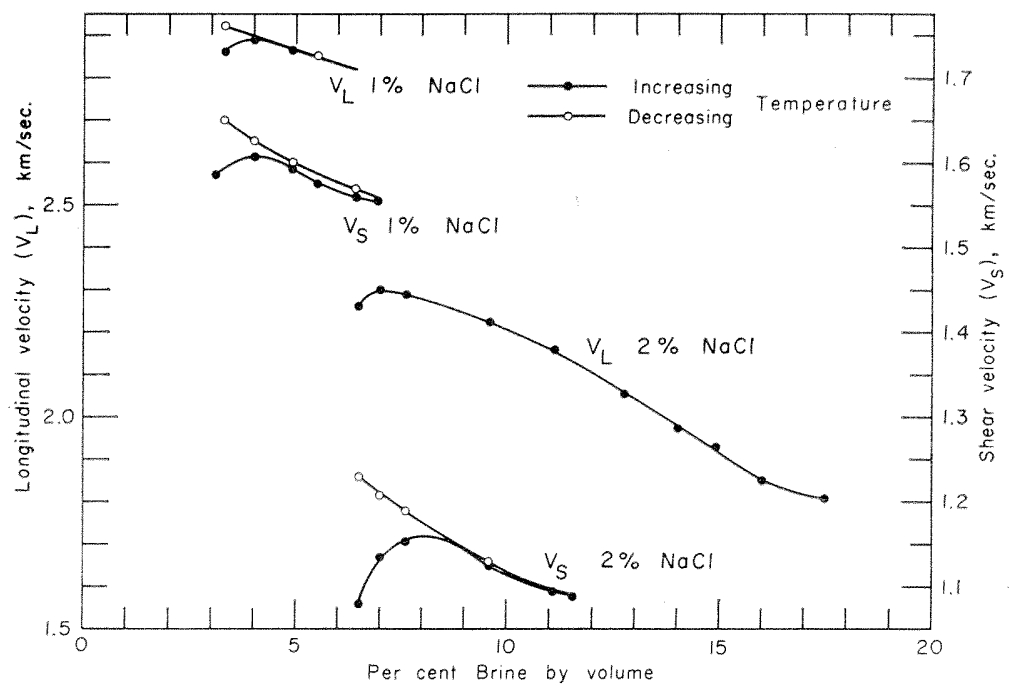

Fig. 5. $V_{L}$ and $V_{S}$ as a function of brine volume. The ice crystals in the $1 \% \mathrm{NaCl}$-ice system have linear dimensions approximately twice as large as in the $2 \% \mathrm{NaCl}$-ice system.

must be much warmer than the ice containing $2 \% \mathrm{NaCl}$. For example, a $7 \%$ brine content occurs at $-8.4^{\circ} \mathrm{C}$ for the ice containing $1 \%$ $\mathrm{NaCl}$ and at $-19^{\circ} \mathrm{C}$ for the ice containing $2 \%$ $\mathrm{NaCl}$. Brine content was computed from the phase diagram in Figure 6 and Weeks [1961]. Contrary to our initial expectations, the velocity is not a unique function of the melt fraction. In the $1 \% \mathrm{NaCl}$ system the velocities decrease by about 1.2 to $1.4 \%$ for each $1 \%$ increment in brine content. In the $2 \% \mathrm{NaCl}$ system, the corresponding decrease in velocity is about $2 \%$.

The $Q$ measurements (Figures 7 through 12) were taken simultaneously with the velocity measurements. The $Q$ decreases slowly with temperature to about $-30^{\circ} \mathrm{C}$ and then begins to rise gradually. There is an abrupt decrease in $Q$ at the eutectic temperature, which in all cases recovers slightly as the temperature is further raised. A peak in anelasticity is often observed at critical points in gas or fluid mixtures. In our case, this phenomenon is complicated by the large change in mechanical properties which occurs when the system goes from a solid-solid to a solid-fluid mixture. It is this mechanical effect that we are primarily interested in. The elastic wave upsets the local thermodynamic equilibrium in a mixed phase region, in this case a salt-water-ice system. This effect is superimposed on the grain boundary loosening associated with the onset of partial melting. When the temperature is reversed, i.e. when the sample is cooled, the ability to supercool (which is related to the difficulty of nucleation) permits nonequilibrium conditions to maintain during the passage of a stress wave, and there is no loss associated with thermodynamic relaxation

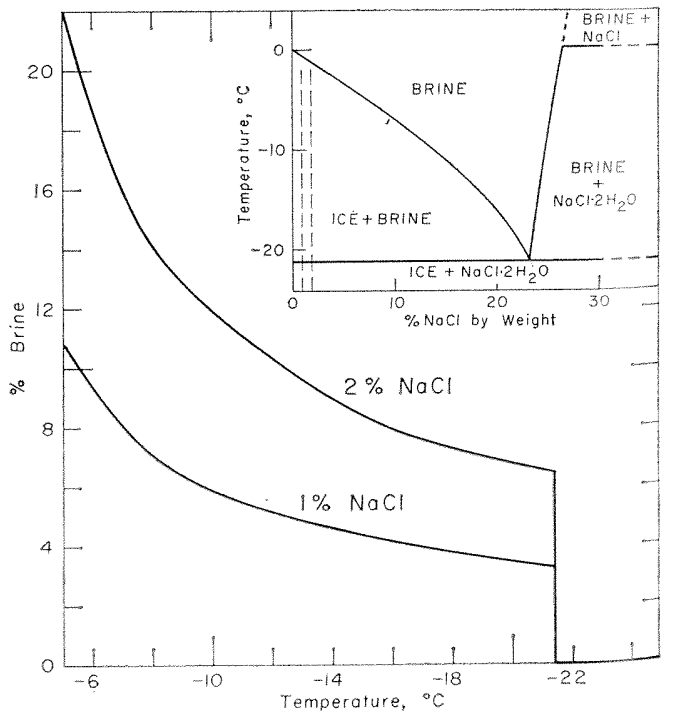

Fig. 6. Volume per cent of brine versus temperature and composition in the system studied. Insert shows the phase diagram of the $\mathrm{NaCl}$ system [Weeks, 1961]. Dashed lines are the compositions studied. 


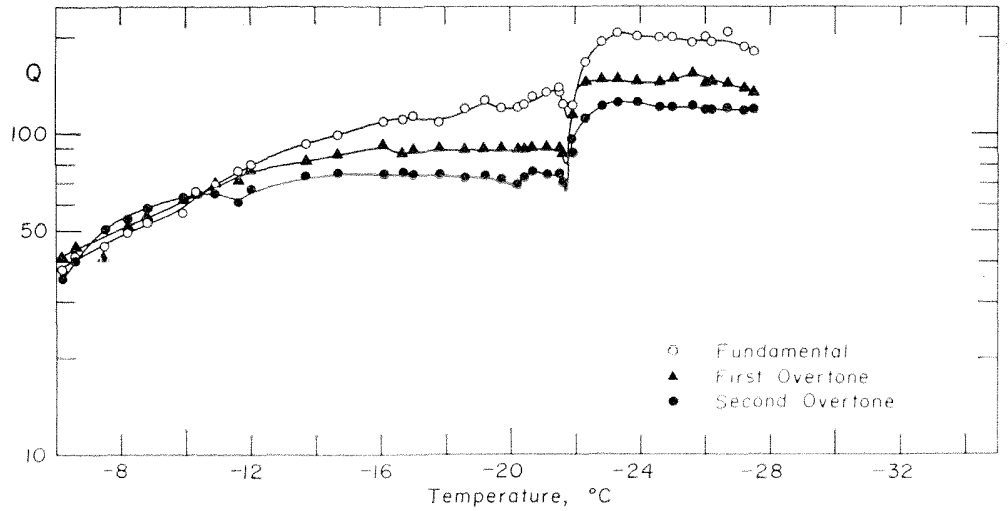

Fig. 7. $Q$ for the first three longitudinal modes or the heating cycle for $1 \% \mathrm{NaCl}$.

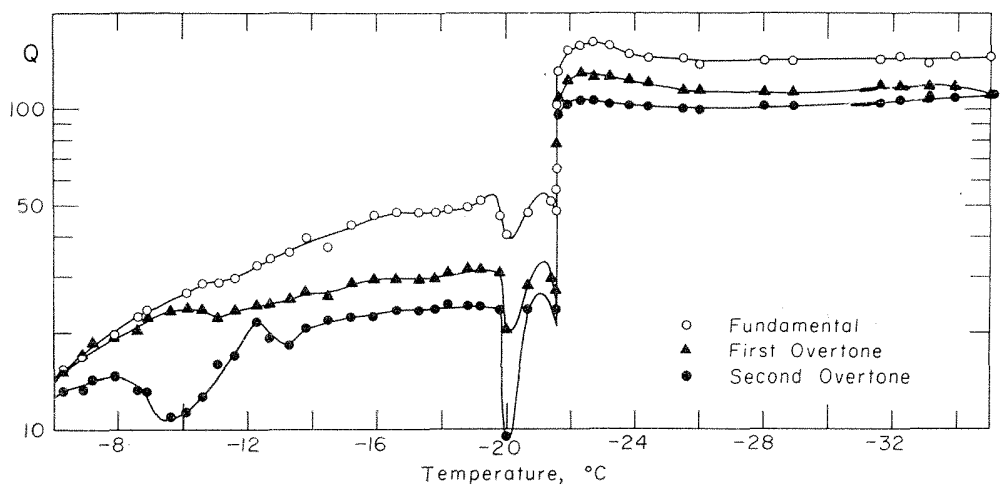

Fig. 8. Q for the first three shear or torsional modes or the heating cycle for $2 \% \mathrm{NaCl}$.

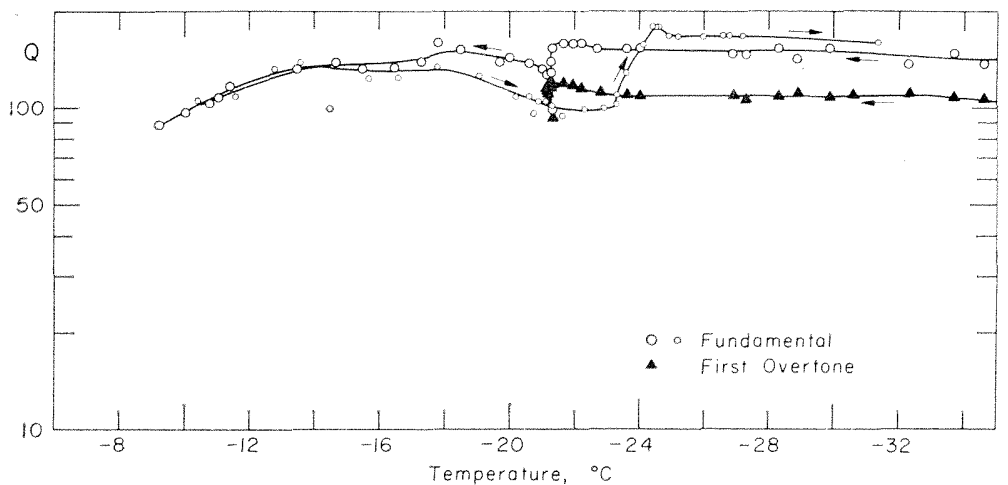

Fig. 9. $Q$ for the first two harmonics, the shear or torsional modes for $1 \% \mathrm{NaCl}$. Arrows indicate whether the data were taken on the warming or cooling cycle.

effects. In this case the losses are associated with mechanical, presumably grain-boundary effects alone. On the warming cycle, the drop in $Q$ at the cutectic point amounted to 48 and $71 \%$ for the fundamental frequency of the longitudi- nal $Q$ 's for the 1 and $2 \% \mathrm{NaCl}$ ice. The corresponding values for the shear $Q$ 's were 37 and $73 \%$.

A further increase in temperature leads to a small increase in $Q$ for the longitudinal modes. 


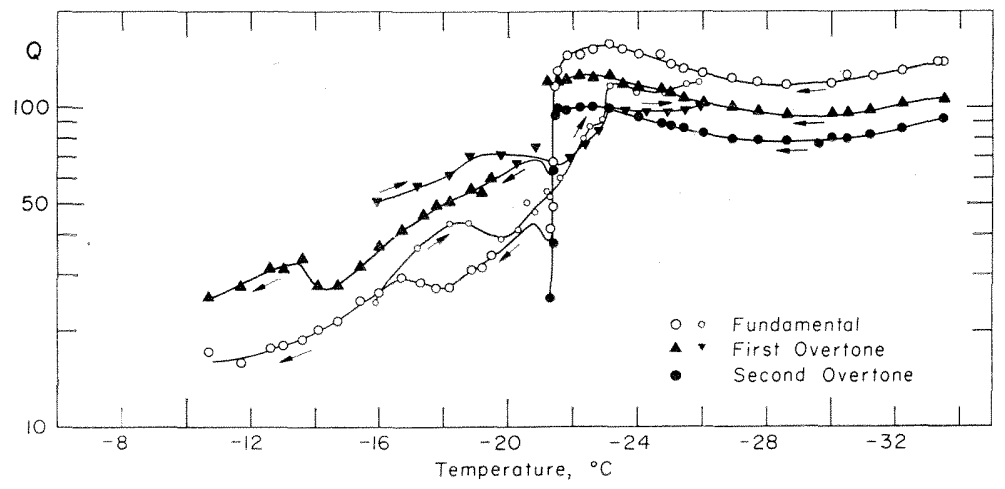

Fig. 10. $Q$ in shear for the first three harmonics for $2 \% \mathrm{NaCl}$. Arrows pointing to the left indicate that the data were taken on the warming cycle. Arrows pointing to the right indicate that the data were taken on the cooling cycle; note the supercooling in the second case.

For the shear modes, especially for the $1 \%$ $\mathrm{NaCl}$ mixture, the increase was very pronounced. At warmer temperatures as the brine content further increases, the $Q$ 's begin to decrease. For comparison, the longitudinal and shear $Q$ 's for pure ice are shown in Figures 11 and 12. For pure ice, and for salt ice below $-35^{\circ} \mathrm{C}, Q$ increases with frequency for the first few harmonics. For higher temperatures in the salt ice, $Q$ decreases as the frequency increases. Above the eutectic temperature this generalization does not hold.

The shear $Q$ data of pure ice show minima for the first three harmonics at $-28^{\circ},-16^{\circ}$, and $-12^{\circ} \mathrm{C}$, respectively. Figure 12 shows the relation of these minima to temperature and relaxation time. The activation energy corresponding to this frequency response is approximately $7.5 \mathrm{kcal} / \mathrm{mole}$.

\section{Size of Ice Platelets and Velocity}

The spacing between the centers of adjacent ice platelets is a linear function of salt concentration and is directly proportional to the square root of the freezing time [Rohatgi and Adams, 1967]. The freezing times for the 1 and $2 \%$ ice rods in this experiment are of the order of 100 to several hundred seconds. This freezing time corresponds approximately to that used in Figure 14 of the above mentioned reference. The spacing between the centers of the platelets of the 1 and $2 \%$ ice is therefore quite similar, and this is confirmed by the photographs described earlier. The other dimensions of the crystals are, however, approximately twice as large for the $1 \%$ ice as for the $2 \%$ ice.

It is clear from Figure 5 that the mechanical properties depend on more than just the brine or melt content. Since most of the brine is con-

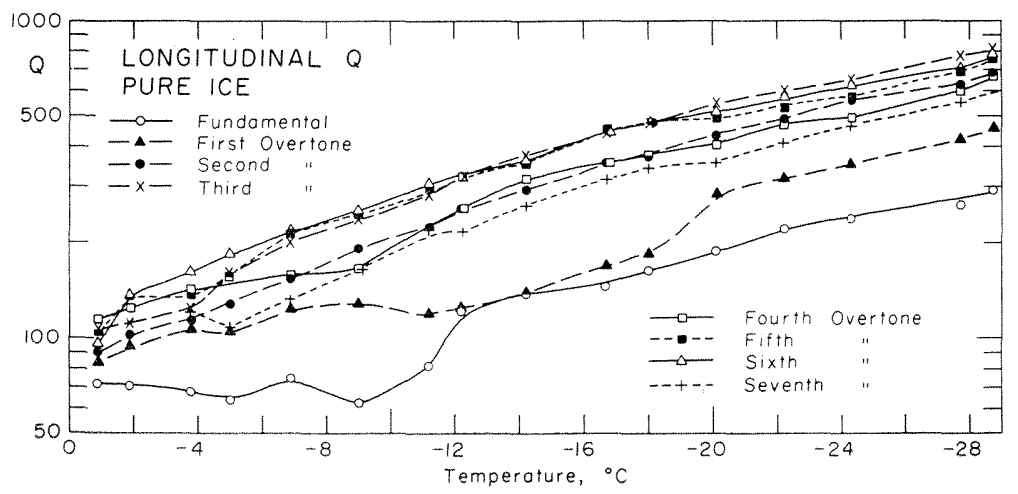

Fig. 11. $Q$ as a function of temperature and frequency for pure ice; longitudinal modes. 

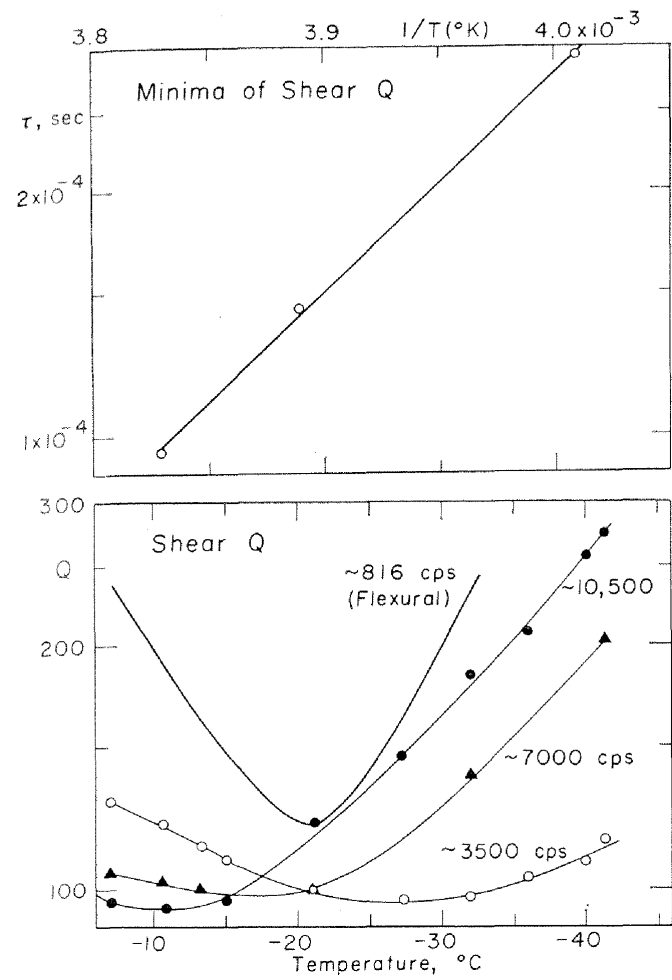

Fig. 12. $Q$ as a function of temperature and hamonic for shear modes in pure ice. Also shown are the results in flexure obtained by Kuroiwa [1965].

tained in layers between subcrystal platelets, the function of the liquid (brine) seems to be one of decoupling at the grain boundaries. The number of grain boundaries per unit volume, an important parameter, is inversely proportional to the surface area of a grain, and the velocity is a function of the brine content times the surface area of the grains.

\section{Conclusions}

There are a number of generalizations that can be made from the data of this investigation. A small volume fraction of liquid has a large effect on the velocity and attenuation of shear and longitudinal waves. As expected, the effect on the shear velocity is considerably more than the effect on the longitudinal velocity. Because of the anisotropy of the ice rods, no direct comparison can be made between the velocities and $Q$ 's of the shear and the longitudinal modes.

The sharp dip in $Q$ at the eutectic point is of more than passing interest. This dip was observed on all the samples but was most pronounced in the shear data for ice containing $1 \%$ $\mathrm{NaCl}$. A multicomponent, multiphase system that is in equilibrium will be disturbed by an acoustical signal. The degree to which the equilibrium is disturbed is a function of the frequency and amplitude of the signal. The rate at which the chemical equilibrium can follow temperature and pressure fluctuations (chemical kinetics) controls the frequency dependence of this part of the ultrasonic absorption. At a given frequency, the absorption is a function of the concentration gradients associated with the temperature and pressure fluctuations caused by the stress wave. Energy is absorbed from the acoustic signal and converted into chemical energy. Some of this chemical energy is released in the form of heat as the system attempts to return to equilibrium. The slopes of the curves in Figure 5 give a measure of the extent to which the equilibrium may be disturbed by a small change in temperature. At the eutectic where the slope is discontinuous, the absorption should be a maximum, as is observed. The above described absorption mechanism is well known and has been used to study reaction kinetics in liquids (see, for example, Tabuchi [1956, 1957]; Yasunaga et al. [1965]; Tatsumoto [1966]) in the megacycles-per-second range.

The preceding arguments suggest that a sharp dip in $Q$ will be associated with the onset of partial melting in the mantle. Solid-solid phase changes may show similar dips. The low-velocity zones of the upper mantle has been interpreted in terms of $(a)$ proximity to the melting point [Press, 1959], (b) high temperature gradients [Gutenberg, 1959; Birch, 1952; Valle, 1956], and $(c)$ chemical inhomogeneity in the mantle [Ringwood, $1962 a$ and $b]$. The data of the present investigation indicate that the seismic velocities and $Q$ values will drop abruptly if the solidus crosses the geotherm. If due to partial melting, the boundaries of the low-velocity zone will be abrupt. This is consistent with recent tectonic and oceanic mantle models. The low velocity zone will also be a zone of high attenuation and this also seems to be the case.

Acknowledgments. It is a pleasure to acknowledge the help of David F. Newbigging who assisted in all phases of the experiment. We are grateful for helpful discussions with Thomas 
Ahrens, Charles Archambeau, and Samuel Epstein.

This research was partially supported by $\mathrm{Na}$ tional Science Foundation grant GA 1003.

\section{ReFERENCES}

Birch, F., Elasticity and constitution of the earth's interior, J. Geophys. Res., 57, 227-286, 1952.

Gutenberg, B., The asthenosphere low-velocity layer, Annali di Geofisica, 12, 439-460, 1959.

Kuroiwa, D., Internal friction of $\mathrm{H}_{2} \mathrm{O}, \mathrm{D}_{2} \mathrm{O}$, and natural glacier ice, U. S. Army Material Command Res. Rept. 131, Cold Regions Research and Engineering Laboratory, New Hampshire, 1965.

Mizutani, H., and H. Kanamori, Variation of elastic wave velocity and attenuative property near the melting temperature, $J$. Phys. Earth, 12(2), 43-49, 1964.

Porkorny, M., Variation of velocity and attenuation of longitudinal waves during the solidliquid transition in Wood's alloy, Studia Geophys. Geodaet. Ceskoslov. Akad. Ved, 9, 1965.

Press, F., Some implications on mantle and crustal structure from $G$ waves and Love waves, J. Geophys. Res., 64, 565-568, 1959.

Ringwood, A. E., A model for the upper mantle, $J$. Geophys. Res., 67, 857-867, 1962a.
Ringwood, A. E., A model for the upper mantle, 2, J. Geophys. Res., 67, 4473-4477, $1962 b$.

Rohatgi, P. K., and C. M. Adams, Jr., Ice-brine dendritic aggregate formed on freezing of aqueous solutions, J. Glaciol., 6, 47, 1967.

Tabuchi, D., Dispersion and absorption of sound in liquids in general chemical equilibrium and its application to chemical kinetics, $J$. Chem. Phys., 26(5), 993-1001, 1956.

Tabuchi, D., Dispersion and absorption of sound in ethyl formate and study of the rotational isomers, J. Chem. Phys., 28(6), 1014-1021, 1957.

Tatsumoto, N., Ultrasonic absorption in propionic acid, J. Chem. Phys., 47(11), 4561-4570, 1966.

Valle, P. E., On the temperature gradient necessary for the formation of a low velocity layer, Ann. Geofis. Rome, 9, 371-377, 1956.

Weeks, W. F., Studies of salt ice, 1, The tensile strength of $\mathrm{NaCl}$ ice, U.S. Army Material Command Research Report 80, U. S. Army Cold Regions Research and Engineering Laboratory, New Hampshire, 1961.

Yasunaga, T., N. Tatsumoto, and M. Miure, Ultrasonic absorption in sodium metaborate solution, J. Chem. Phys., 43(8), 2735-2738, 1965.

(Received April 8, 1968; revised June 7 , 1968.) 